\title{
Merkel Cell Carcinoma in a Cat
}

\author{
Kiyokazu OZAKI ${ }^{1) *}$ and Isao NARAMA ${ }^{1)}$ \\ ${ }^{1)}$ Department of Pathology, Faculty of Pharmaceutical Science, Setsunan University, 45-1 Nagaotohge-cho, Hirakata, Osaka 573-0101, \\ Japan
}

(Received 5 February 2009/Accepted 30 March 2009)

ABSTRACT. A 17-year-old spayed female Japanese domestic cat presented with a solitary cutaneous mass in the right thoracic area. Histopathologically, the mass consisted mainly of round tumor cells that had infiltrated throughout the dermis and deep subcutaneous tissue. The proliferating pattern of tumor cells was solid but also trabecular or cord-like in some areas, and lined with small cells resembling mature lymphocytes or basal cells. The tumor cells were positive for cytokeratin 20 and synaptophysin. The positive reaction for cytokeratin 20 showed localized to diffuse pattern. This is the first report describing now cytokeratin 20 was clearly beneficial for the differential diagnosis of feline Merkel cell carcinoma.

KEY WORDS: cytokeratin 20, feline, Merkel cell carcinoma, neuroendocrine tumor.

J. Vet. Med. Sci. 71(8): 1093-1096, 2009

Merkel cell carcinoma is a cutaneous neuroendocrine tumor, which is a very rare cutaneous tumor in dogs and cats $[1,12]$. Histopathologically, it is composed of relatively uniform small round cells frequently arranged in a sheetlike, cord-like or trabecular pattern. Therefore, it might be quite difficult to distinguish from trichoblastoma or sweat gland tumor by the morphological character alone. The diagnostic value of cytokeratin 20 immunostaining in human Merkel cell carcinoma has attracted an increasing attention, since its characteristic perinuclear dot-like staining pattern is specific to those tumor cells only [11]. In feline skin, cytokeratin 20 reacted only with solitary basal cells of the cutaneous epithelium, identified as Merkel cells because of the round morphology and basal location, and with acini and ducts of apocrine glands and acini of eccrine glands [5]. Since the occurrence of Merkel cell carcinoma in animals is so very rare, the usefulness and staining pattern of immunocytochemical examinations using cytokeratin 20 had not been reported. This is the first report of a case of feline Merkel cell carcinoma with diagnostic confirmation by immunocytochemical staining by cytokeratin 20 .

A 17-year-old spayed female Japanese domestic cat was presented with a solitary cutaneous mass in the right thoracic area (Fig. 1). At clinical examination, a solitary discshaped, well-circumscribed nodule was observed; no other nodular lesion was seen. The cutaneous mass and superficial cervical lymph node were resected surgically. Five months later the cat died, but no necropsy was performed. Grossly, the disc-shaped nodule was $30 \times 30 \times 5 \mathrm{~mm}$ in size, and part of the overlying skin was ulcered. The cut surface of the mass was solid and white in color. Specimens were fixed in $10 \%$ neutral formalin $(\mathrm{pH} \mathrm{7.4)}$, dehydrated in a graded series of ethanol, and embedded in paraffin, and 4$\mu \mathrm{m}$-thick sections were stained with hematoxylin and eosin.

\footnotetext{
* Correspondence to: Ozaki, K., Department of Pathology, Faculty of Pharmaceutical Science, Setsunan University, 45-1 Nagaotohge-cho, Hirakata, Osaka 573-0101, Japan. e-mail: ozaki@pharm.setsunan.ac.jp
}

Grimelius reaction was performed to evaluate neuroendocrine granules. Immunohistochemical staining was performed by a labeled-polymer method using N-Histofine MAX PO (M or R) (Nichirei, Japan). The primary antibodies used for each section were anti-human CD3 (diluted at $1: 100$, rabbit polyclonal antibody, DAKO, Denmark, T cells of normal lymph node were used as a positive control), antihuman CD20 (diluted at 1:400, rabbit polyclonal antibody, Lab Vision, U.S.A., B cells of normal lymph node were used as a positive control), anti-human CD79acy (diluted at $1: 25$, monoclonal antibody HM57, DAKO, B cells of normal lymph node were used as a positive control), antihuman cytokeratin 8/18 (diluted at 1:50, mouse monoclonal antibody, Novocastra, UK, apocrine sweat gland cells of this skin were used as a positive control), anti-human cytokeratin 20 (diluted at 1:20, mouse monoclonal antibody, DAKO, apocrine sweat gland cells of this skin were used as a positive control), anti-human cytokeratin (MNF116) (diluted at 1:100, mouse monoclonal antibody MNF116, DAKO, normal epidermis of this skin were used as a positive control), anti-cytokeratin (CAM5.2) (prediluted, mouse monoclonal antibody CAM5.2, Becton, apocrine sweat gland cells of this skin were used as a positive control), anticytokeratin (AE1/AE3) (diluted at 1:100, mouse monoclonal antibody AE1/AE3, DAKO, normal epidermis of this skin were used as a positive control), anti-human chromogranin A (diluted at 1:100, mouse monoclonal antibody, DAKO, medulla cells of normal adrenal gland were used as a positive control), anti-human synaptophysin (diluted at 1:100, rabbit polyclonal antibody, DAKO, medulla cells of normal adrenal gland were used as a positive control), antithyroid transcription factor (diluted at 1:50, mouse monoclonal antibody, DAKO, pulmonary carcinoma were used as a positive control), and anti-PGP 9.5 (diluted at 1:200, rabbit polyclonal antibody, DAKO, axon of this skin were used as a positive control).

Histopathologically, the mass consisted mainly of round tumor cells infiltrated throughout the dermis and deep sub- 

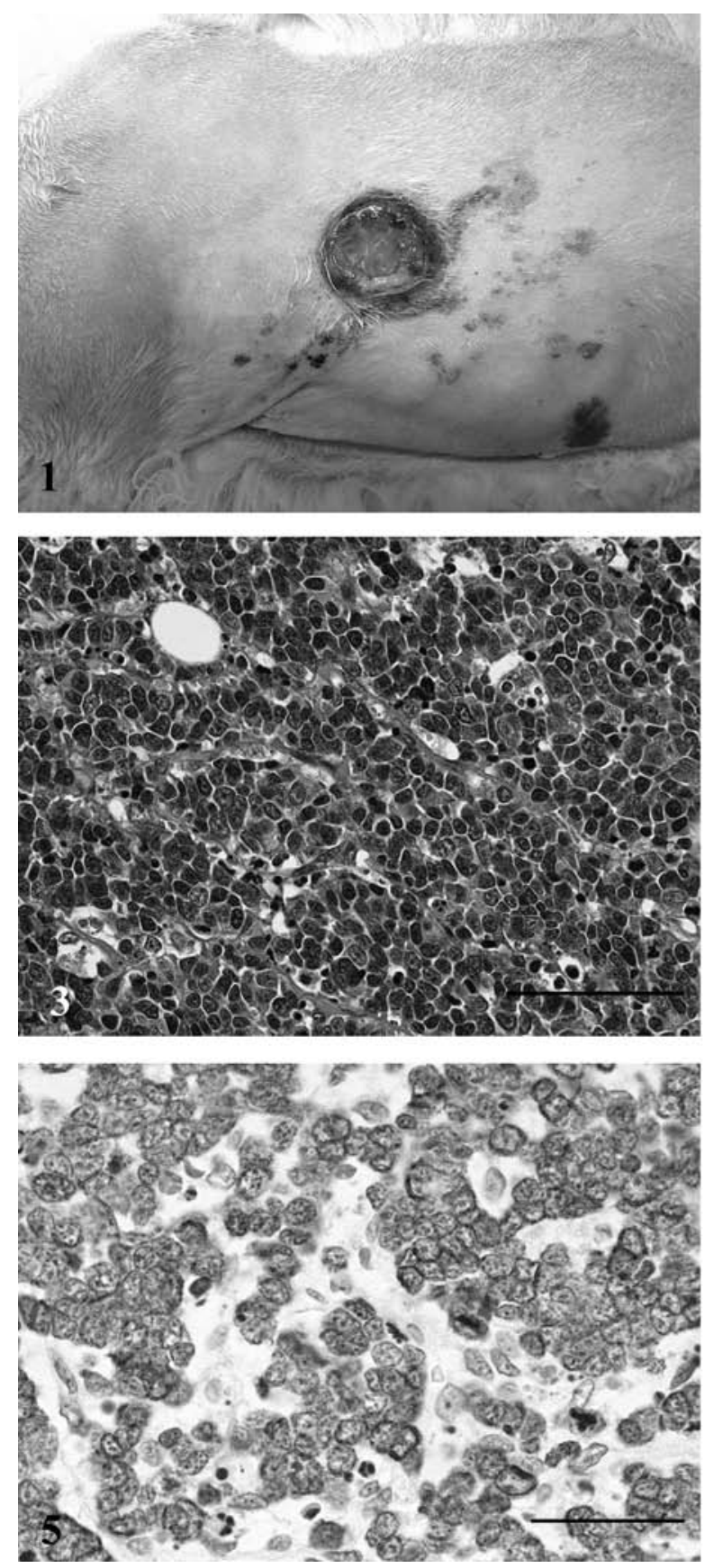

cutaneous tissue near the cutaneous muscle layer. These cells had proliferated from the superficial layer to the deep dermis, but had not invaded the epidermal layer of the skin and hair follicles. The proliferating pattern of tumor cells was solid, but also occasionally trabecular or cord-like in some areas (Fig. 2). Occasionally, the tumor cells were arranged to form a cord- or gland-like structures with rare intracytoplasmic vaculoles in some areas. Their size varied considerably from small cells resembling mature lymphocytes or basal cells to large cells with polygonal nuclei up to 3 times of the small ones. The cytoplasm of many tumor
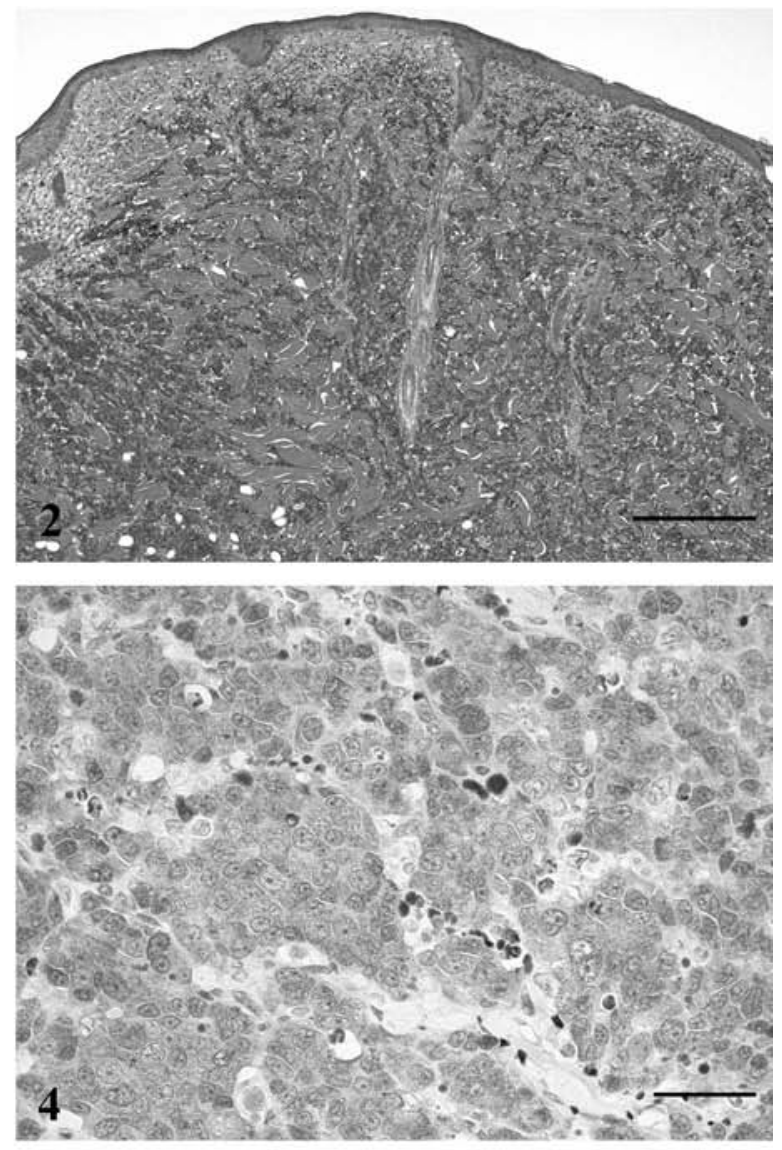

Fig. 1. A solitary disc-shaped, well-circumscribed nodule on right thoracic area with central ulceration.

Fig. 2. The proliferating pattern of tumor cells was solid but also trabecular or cord-like in some areas. HE. Bar $=500 \mu \mathrm{m}$.

Fig. 3. The cytoplasm of tumor cells is scanty but small amounts of cytoplasmic rims were also visible in many cells. HE. $\mathrm{Bar}=50 \mu \mathrm{m}$.

Fig. 4. Immunoreactivity for synaptophysin is detected. Almost all tumor cells are positive. Bar $=50 \mu \mathrm{m}$.

Fig. 5. Immunoreactivity to cytokeratin 20 is detected. The positivity for cytokeratin 20 has localized to diffuse pattern. Bar $=50 \mu \mathrm{m}$.

cells was scanty, though small amounts of cytoplasmic rims were also frequently visible (Fig. 3). Many nuclei contained fine granular chromatin and multiple small nucleoli, and mitotic figures and apoptotic cells were frequent. Necrotic areas were often observed in the tumor mass and a large necrotic area extended from dermo-epidermal junction to the deep dermis. Metastasis to the lymph node was also seen.

The Grimelius reaction was positive in tumor cells, as evidenced by small dark granules in the cytoplasm. Immunohistochemically, almost all the tumor cells were positive 
for cytokeratin 20, CAM5.2 and synaptophysin (Fig. 4). The positivity for cytokeratin 20 and CAM5.2 had assumed localized to diffuse pattern (Fig. 5). The cells showed focal reactivity for chromogranin A and PGP9.5, but no positive reaction was observed for cytokeratin $8 / 18$, cytokeratin MNF116, AE1/AE3, CD3, CD20, CD79, or thyroid transcription factor.

Both the light and immunohistochemical findings in this tumor supported a diagnosis of Merkel cell carcinoma. Differential diagnoses included lymphoma, plasmacytoma, trichoblastoma, sweat gland tumor and metastatic neuroendocrine tumor. In contrast to the round nuclei with dense chromatin and the large distinct nucleoli and scanty cytoplasm of the tumor cells characteristic of the malignant lymphoma, cells in the present case were characterized by scanty but constantly visible cytoplasm, and round nuclei with small multiple nucleoli and fine granular chromatin. In addition, the cells in our case often also exhibited trabecular or cord-like pattern, suggesting an epithelial origin in some areas. Immunohistochemically negative staining for $\mathrm{CD} 3$, CD20 and CD79 provided the most definitive evidence needed to distinguish them from malignant lymphoma including plasmacytoma.

Some kinds of trichoblastoma are difficult to differentiate from Merkel cell carcinoma [2, 8]. Trichoblastoma often included focal positivity for synaptophysin, chromogranin A and cytokeratin 20, indicating neuroendocrine differentiation $[4,13]$. However, in the present case, many tumor cells were positive for synaptophysin, and focal positivity for Grimelius reaction and chromogranin A were seen. These were specific markers of neuroendocrine differentiation. In addition, PGP9.5 was focally positive in our case. Expression of PGP9.5 is highly specific to neurons and to cells of neuroendocrine system and their tumors, but some authors claimed that PGP 9.5 is a specific marker of neural differentiation in tumor [3]. It was unclear that positivity for PGP9.5 in our case showed neuroendocrine differentiation, but expression of three neuroendocrine markers except PGP9.5 was confirmed that our case was derived from neuroendocrine cells. In the present case, the gland-like structure and intracytoplasmic cysts of these cells with positive staining for CAM5.2 suggested sweat glands as their origin. However, negative staining for cytokeratin $8 / 18$ served to exclude sweat gland differentiation. Moreover, the morphologic pattern of immunohistochemical positivity for cytokeratin 20 was very characteristic and indicative of Merkel cell tumor [10]. It has been reported that an immunohistochemical examination using cytokeratin 20 is extremely useful in distinguishing Merkel cell tumors from trichoblastoma and sweat gland tumors [11]. Merkel cell tumors are characterized by perinuclear dot-like structures (keratin button) for cytokeratin $20[10,11]$, but these pattern is not universally seen as is true of those in the present case [7].

In humans, small cell carcinoma of the lung often metastasizes to the skin [6]. The growth pattern of that tumor resembles that of a Merkel cell tumor. Cytokeratin 20 was usually negative while thyroid transcription factor was pos- itive in such small cell carcinomas, but there has been no equivalent report on animals [6,9-11]. In our case, as necropsy was not performed, a metastatic neuroendocrine tumor including small cell carcinoma of lung, could not be completely excluded. However, negative for thyroid transcription factor- 1 and positive for cytokeratin 20 in our case was indicated to assist in the differentiation of Merkel cell carcinoma from metastatic small cell carcinoma of the lung.

Only two other cases of Merkel cell carcinoma in cats have been reported $[1,12]$. One case showed recurrent tumor growth and pulmonary metastasis, but no clinical signs of a malignant nature were observed in the other case. The finding of lymph node metastasis together with many mitotic figures in our case suggested a malignant character of the tumor. On the other hand, Merkel cell tumors seemed to be benign in dogs [8], while most were malignant in humans. Therefore, the clinical behavior of this tumor in cats was shown to be similar to that in human.

\section{REFERENCES}

1. Bagnasco, G., Properzi, R., Porto, R., Nardini, V., Poli, A. and Abramo, F. 2003. Feline cutaneous neuroendocrine carcinoma (Merkel cell tumor): clinical and pathological findings. Vet. Dermatol. 14: 111-115.

2. Ball, N.J. and Tanhuanco-Kho, G. 2007. Merkel cell carcinoma frequently shows histologic features of basal cell carcinoma: a study of 30 cases. J. Cutan. Pathol. 34: 612-619.

3. Campbell, L.K., Thomas, J.R., Lamps, L.W., Smoller, B.R. and Folpe, A.L. 2003. Protein gene product 9.5 (PGP 9.5) is not a specific marker of neural and nerve sheath tumors: an immunohistochemical study of 95 mesenchymal neoplasms. Mod. Pathol. 16: 963-969.

4. Collina, G., Eusebi, V., Capella, C. and Rosai, J. 1998. Merkel cell differentiation in trichoblastoma. Virchows Arch. 433: 291-296.

5. Espinosa de los Monteros, A., Fernandez, A., Millan, M.Y., Rodriguez, F., Herraez, P. and Martin de las Mulas, J. 1999. Coordinate expression of cytokeratins 7 and 20 in feline and canine carcinomas. Vet. Pathol. 36: 179-190.

6. Hanly, A.J., Elgart, G.W., Jorda, M., Smith, J. and Nadji, M. 2000. Analysis of thyroid transcription factor- 1 and cytokeratin 20 separates merkel cell carcinoma from small cell carcinoma of lung. J. Cutan. Pathol. 27: 118-120.

7. Jensen, K., Kohler, S. and Rouse, R.V. 2000. Cytokeratin staining in Merkel cell carcinoma: an immunohistochemical study of cytokeratins 5/6, 7, 17, and 20. Appl. Immunohistochem Mol. Morphol. 8: 310-315.

8. Konno, A., Nagata, M. and Nanko, H. 1998. Immunohistochemical diagnosis of a Merkel cell tumor in a dog. Vet. Pathol. 35: 538-540.

9. Lin, X., Saad, R.S., Luckasevic, T.M., Silverman, J.F. and Liu, Y. 2007. Diagnostic value of CDX-2 and TTF-1 expressions in separating metastatic neuroendocrine neoplasms of unknown origin. Appl. Immunohistochem Mol. Morphol. 15: 407-414.

10. Metz, K.A., Jacob, M., Schmidt, U., Steuhl, K.P. and Leder, L.D. 1998. Merkel cell carcinoma of the eyelid: histological and immunohistochemical features with special respect to differential diagnosis. Graefes Arch. Clin. Exp. Ophthalmol. 236: 561-566. 
11. Moll, R., Lowe, A., Laufer, J. and Franke, W.W. 1992. Cytokeratin 20 in human carcinomas. A new histodiagnostic marker detected by monoclonal antibodies. Am. J. Pathol. 140: 427447.

12. Patnaik, A.K., Post, G.S. and Erlandson, R.A. 2001. Clinicopathologic and electron microscopic study of cutaneous neuroendocrine (Merkel cell) carcinoma in a cat with comparisons to human and canine tumors. Vet. Pathol. 38: 553-556.

13. Wiedemeyer, K. and Hartschuh, W. 2009. Trichoblastomas with Merkel cell proliferation in nevi sebacei in Schimmelpenning-Feuerstein-Mims syndrome--Histological differentiation between trichoblastomas and basal cell carcinomas. J. Dtsch. Dermatol. Ges. 7: 612-614. 